\title{
Transillumination imaging through biological tissue by single-pixel detection
}

\author{
Vicente Durán, ${ }^{a}$ Fernando Soldevila, ${ }^{b}$ Esther Irles ${ }^{b}$ Pere Clemente,${ }^{b}$ Enrique Tajahuerce, ${ }^{b}$ \\ Pedro Andrés ${ }^{c}$ and Jesús Lancis ${ }^{b}$ \\ ${ }^{a}$ Microtechnology and Nanoscience Department (MC2), Chalmers University of Technology, \\ SE 41296, Gothenburg, Sweden; \\ ${ }^{b}$ GROC.UJI, Institute of New Imaging Technologies, Universitat Jaume I, 12070 Castelló, \\ Spain; \\ ${ }^{c}$ Departamento de Óptica, Universitat de València, 46100 Burjassot, Spain;
}

\begin{abstract}
One challenge that has long held the attention of scientists is that of clearly seeing objects hidden by turbid media, as smoke, fog or biological tissue, which has major implications in fields such as remote sensing or early diagnosis of diseases. Here, we combine structured incoherent illumination and bucket detection for imaging an absorbing object completely embedded in a scattering medium. A sequence of low-intensity microstructured light patterns is launched onto the object, whose image is accurately reconstructed through the light fluctuations measured by a single-pixel detector. Our technique is noninvasive, does not require coherent sources, raster scanning nor time-gated detection and benefits from the compressive sensing strategy. As a proof of concept, we experimentally retrieve the image of a transilluminated target both sandwiched between two holographic diffusers and embedded in a $6 \mathrm{~mm}$-thick sample of chicken breast.
\end{abstract}

Keywords: Computational Imaging, Compresssive Sensing, Scattering Media

\section{INTRODUCTION}

Optical methods are growing into an essential tool in biomedical science. They are noninvasive, fast, cost-efficient and, unlike techniques based on ionizing radiation, they do not pose a health risk. In the realm of diagnosis, we have witnessed during the past few years how optical imaging has assisted clinicians in the detection and evaluation of suspicious lesions. However, the "elephant in the room" question that should be addressed is the short penetration depth of light into tissue, in contrast to ultrasound or X-ray technologies. Photons propagating through an inhomogeneous medium can experience a certain number of scattering events and, as a result, the emerging radiation is a mix of two components: "image-bearing" photons, travelling nearly in the incoming direction, and diffusive photons, which have experienced multiple scattering inside the medium and follow a completely uncorrelated direction at the output. The intensity of the unscattered light component falls off with the medium thickness. As a consequence, imaging begins to be difficult or unfeasible at a penetration depth larger than the tissue transport mean free path, a measurement of the mean distance that photons travel before they completely 'forget' their initial direction. ${ }^{1}$ Imaging techniques that model the random propagation of diffuse photons, such as multispectral optoacoustic tomography ${ }^{2}$ or hybrid fluorescence molecular tomography, ${ }^{3}$ can reach greater penetration depths $(>1 \mathrm{~cm}$ in tissue), but they only enable macroscopic imaging due to their relatively low resolution. ${ }^{4}$ Recently it has been demonstrated a non-invasive approach that uses diffuse photons while preserving optical resolution. This method is based on the angular correlation ("memory effect") inherent to the speckle patterns generated by photon scattering. ${ }^{5}$ However, current implementations are restricted to thin scattering media, ${ }^{6}$ since the memory-effect range is inversely proportional to the medium thickness.

In this paper we present a novel technique able to image objects embedded in scattering media. This "scattering-free" imaging approach is based on the concept of the single-pixel camera ${ }^{7}$. Our incoherent lowintensity technique uses "image-bearing" photons at the illumination stage, but takes advantage of the total flux of the photons at the detection step. We capture the object information with a "bucket" detector without spatial resolution, responsible for measuring light fluctuations that are correlated with a set of light patterns sequentially

Optical Coherence Imaging Techniques and Imaging in Scattering Media, edited by

Brett E. Bouma, Maciej Wojtkowski, Proc. of SPIE-OSA Biomedical Optics, SPIE Vol. 9541

95410B · ( 2015 SPIE-OSA · CCC code: 1605-7422/15/\$18 · doi: 10.1117/12.2183457

Proc. of SPIE-OSA Vol. 9541 95410B-1 
projected onto the object. The single-pixel detection of diffusive photons has demonstrated to be crucial for image transmission through scattering media, even when they are dynamic. ${ }^{8}$ However, in our previous experiments we considered an illumination stage that was free of scattering. Here, we overcome this strong limitation, demonstrating non-invasive imaging of an absorbing object completely embedded in an inhomogeneous medium. Such an advance represents a significant step towards the use of single-pixel imaging in more realistic scenarios, especially in biomedical applications.

As a proof of concept, we present experimental results for an object hidden by a couple of holographic diffusers. To test the potentiality of our approach for imaging in tissue, the above diffusers are replaced by two $3 \mathrm{~mm}$-thick layers of chicken breast. For such a tissue thickness, multiple scattering is the predominant effect. Image reconstructions inside the "diagnostic window" (from $650 \mathrm{~nm}$ to $1000 \mathrm{~nm}$ ) are presented.

\section{METHOD AND RESULTS}

We used the experimental setup shown schematically in Fig. 1. Spatially and temporally incoherent light coming from a white-light source impinges onto a digital micromirror device (DMD). The DMD (DLP Discovery 4200, Texas Instruments) produces a set of illumination patterns, which are projected by an optical relay system onto a high-contrast object embedded in a non-absorbing inhomogeneous medium. The unit cell of the binary intensity patterns displayed onto the DMD was composed of $4 \times 4$ DMD pixels and the length of each pixel is $13.7 \mu \mathrm{m}$. Two thin lenses projected the illuminating patterns onto the object and enlarged the unit cell length to $148 \mu \mathrm{m}$.

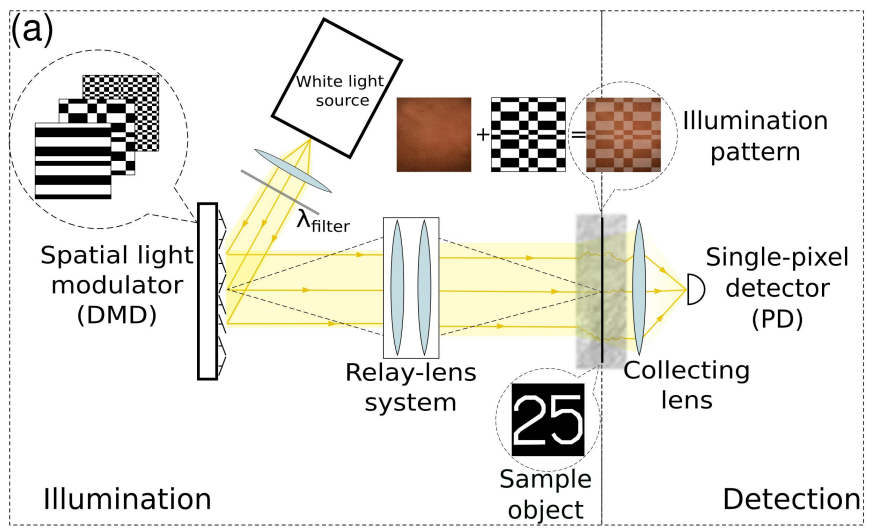

(b)

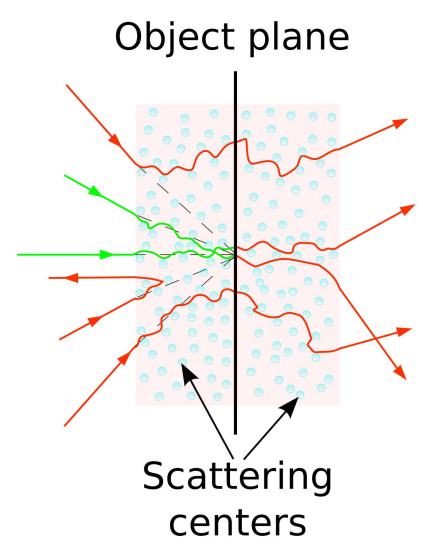

Figure 1. Operation principle. (a), Schematic of the optical setup. We can see: examples of projected patterns (upper left inset); weighted superposition of the diffuse background and the illumination pattern, artificially increased to make it visible (upper right inset); and binary amplitude object (lower inset). (b), A ray light representation corresponding to a bright point of an illumination pattern through an object embedded in a turbid medium. 
The light that hits the object consists of two superimposed components: a strong diffuse halo plus an "imagebearing" weak signal. As can be seen at Fig. 1 (b), for a medium that produces multiple scattering, photons are randomly deviated from the directions they would trace in a homogeneous medium (dashed lines). Those diffuse light rays are red colored in our scheme. This forms a diffuse halo on the object plane, as rays arrive at multiple positions instead of the desired one. However, a fraction of the incoming rays follows a zigzagging path close to the dashed one, even after several interactions with the scattering centres. Those quasi ballistic or snake rays are green colored in our scheme. If such rays are transmitted by the object, they eventually become diffuse rays as a consequence of the second scattering process. Finally, in our transillumination geometry, the light emerging in any direction from the back inhomogeneous layer is concentrated by an optical collecting system formed by two condenser lenses. This system ensured that the light was focused within the sensor area $\left(13 \mathrm{~mm}^{2}\right)$ of a photodiode (DET36A Si Biased Detector, Thorlabs). An analog-to-digital converter (NI USB-6003, National Instruments) digitized the photodetected signal. Custom software written in LabVIEW was used to control the measurement process.

To assess the capability of our technique for imaging through scattering media, we retrieved an image of a binary amplitude object sandwiched between two thin holographic diffusers (Edmund Optics \#T48-002 and \#T54-497). The object was a square part of a resolution target (NBS 1963A) with a size of about $1 \mathrm{~cm}$. The projected binary amplitude patterns were a shifted and rescaled version of the Walsh-Hadamard matrices. The number of illumination patterns was the same as the number of pixels of the recovered image (4096 patterns, $64 \times$ 64 pixels). We changed the photodiode and collecting lens to obtain the conventional image with a photographic objective and a digital camera (UI-3480CP-M, Imaging Developmetn Systems). Both conventional and single-

(a)

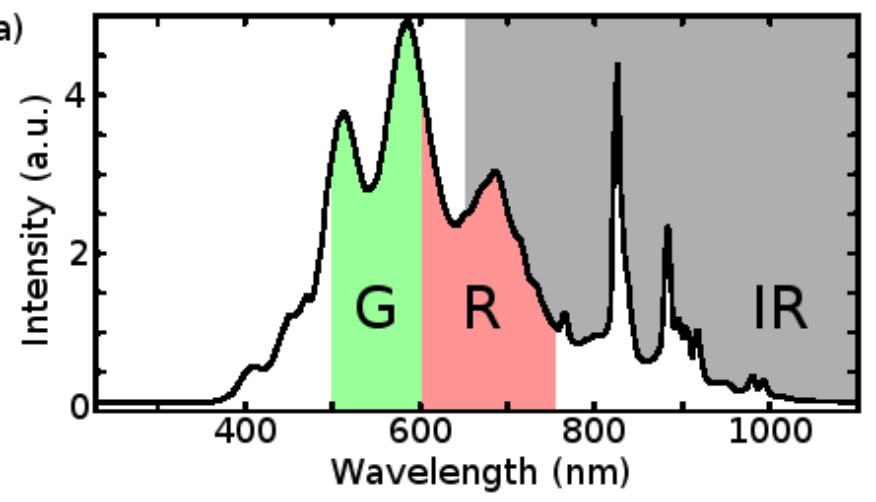

(b)

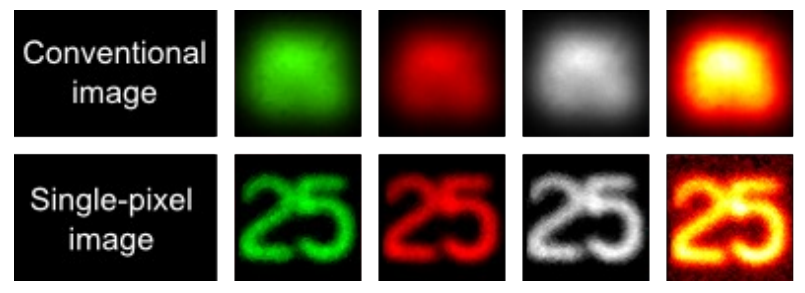

Figure 2. (a), Power spectrum of the Xe lamp used in the experiments. In order to select different spectral bands, several combinations of dichroic filters were used. (b), Set of pictures captured both with a traditional camera and the single-pixel configuration when the object is sandwiched between two holographic diffusing layers. The pseudocolored picture (last column) corresponds to the full spectrum image (without filters). The other pictures show the results in the green, red and infrared bands, respectively.

pixel pictures are presented in Fig. 2. We used a set of dichroic filters to select three spectral bands in the green, red and infrared at the spectrum of our Xe lamp, see Fig. 2(a). Even though the conventional images have a higher resolution, the halo originated by the thin diffusors makes it impossible to recover any spatial information about the scene. Meanwhile, the single-pixel approach allows us to recover the object information. We also studied the spectral behaviour of the diffusers. As can be seen in Fig. 2(b), our technique is able to recover 
information in all three bands with very similar quality. As a consequence, it can work with the full spectrum given by the light source.

Once the method had been proved to work with holographic diffusers, we also tried to recover and object embedded in a volume diffuser. In this experiment, we chose chicken breast tissue as biological scattering medium. The results can be seen in Fig. 3. A reference image for the object is shown in Fig. 3(a). The object was sandwiched between two layers of chicken breast, with a thickness of $2.84 \mathrm{~mm}$ and $2.92 \mathrm{~mm}$, respectively (Fig. 3(b)). No chemical or mechanical processes were applied to the tissue layers. They were placed between two transparent plastic films in order to fix them to the object under study. The image reconstruction is presented in Fig. 3(c). A sample of the measured light fluctuations, corresponding to the first 500 Hadamard patterns, is shown in Fig. 3(d). Such fluctuations make it possible to reconstruct the object image, as can be observed by comparing them with those obtained with the object immersed in air.
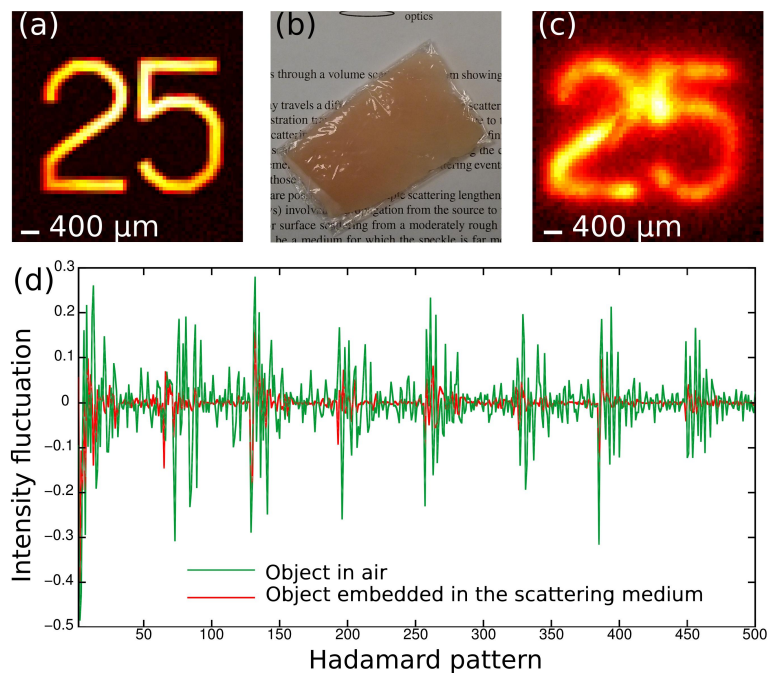

Figure 3. Imaging inside a biological tissue: experimental results. (a), Single-pixel reconstruction of the binary amplitude object. For this reconstruction, the object was immersed in air. (b), Photograph of one of the 3-mm samples of chicken breast that were used in this experiment. (c), Image reconstruction $(64 \times 64$ pixels $)$ when the object was embedded in chicken breast. (d), Normalized intensity fluctuations corresponding to the first 500 Hadamard patterns used to recover the object in (a) and (c).
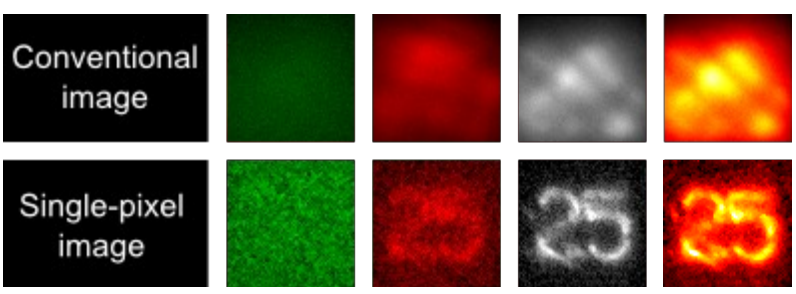

Figure 4. Spectral study of a biological tissue. From the left to the right column: images for green, red and infrared bands. The reconstruction for the full source spectrum is also shown in the last column (pseudocolored image).

The ability of our technique for imaging through tissue along the light source spectrum is analyzed in Fig. 4. In this case, the object was placed between two chicken samples with a thickness of $2.4 \mathrm{~mm}$ and $2.9 \mathrm{~mm}$, respectively. In thick biological tissues photons undergo multiple scattering as they migrate through the medium. This multiple scattering mechanism is quite different from that observed in the thin holographic diffusers. Now, the reduced scattering parameter (the inverse of the transport mean free path) varies across the spectrum. For chicken breast, it decreases smoothly with increase in wavelength. ${ }^{9}$ As a consequence, the retrieved images 
degrade progressively towards shorter wavelengths of the visible spectrum, as can be observed in Fig. 4. For the millimeter tissue thickness that we are considering here, multiple scattering dominates over optical absorption, which means that a collimated beam impinging onto the tissue is basically scattered out of the incident light direction. The improvement of image reconstructions from $650 \mathrm{~nm}$ shows the potentiality of our approach for imaging in the tissue optical window $(650-1350 \mathrm{~nm})$, where light reaches its maximum penetration depth. ${ }^{1}$

\section{CONCLUSIONS}

In conclusion, we have presented a technique that merges Hadamard illumination and single-pixel photodetection for non-invasive imaging through scattering media. In our approach, images free of scattering noise are reconstructed from the correlation between a set of illumination patterns and the corresponding intensity fluctuations, which are measured by a "bucket" detector. We have obtained results for an absorbing object sandwiched between two opaque scattering layers, as well as for the same object embedded in a millimeter sample of chicken breast tissue, where the light experienced multiple scattering. The spectral behavior of the scattering medium has been analyzed in both cases, demonstrating imaging through tissue slides inside the "therapeutic window". The above goals have been reached without increasing the cost and complexity of the imaging system, as is evidenced by the use of a white-light lamp and an off-the-shelf DMD. In our optical scheme, image resolution and field of view are controlled by the DMD parameters and the data acquisition process can be sped up by the application of compressive sensing. ${ }^{10}$ In addition, the freedom to select a proper bucket detector could lead to performing hyperspectral or polarimetric imaging even in the presence of scattering media. ${ }^{11}$

\section{REFERENCES}

[1] Tuchin, V., [Tissue Optics], SPIE, 1000 20th Street, Bellingham, WA 98227-0010 USA (Sept. 2007).

[2] Razansky, D., Distel, M., Vinegoni, C., Ma, R., Perrimon, N., Köster, R. W., and Ntziachristos, V., "Multispectral opto-acoustic tomography of deep-seated fluorescent proteins in vivo," Nature Photonics 3, 412-417 (June 2009).

[3] Vinegoni, C., Pitsouli, C., Razansky, D., Perrimon, N., and Ntziachristos, V., "In vivo imaging of Drosophila melanogaster pupae with mesoscopic fluorescence tomography.," Nature methods 5, 45-7 (Jan. 2008).

[4] Ntziachristos, V., Ripoll, J., Wang, L. V., and Weissleder, R., "Looking and listening to light: the evolution of whole-body photonic imaging.," Nature biotechnology 23(3), 313-320 (2005).

[5] Bertolotti, J., van Putten, E. G., Blum, C., Lagendijk, A., Vos, W. L., and Mosk, A. P., "Non-invasive imaging through opaque scattering layers.," Nature 491, 232-234 (Nov. 2012).

[6] Katz, O., Heidmann, P., Fink, M., and Gigan, S., "Non-invasive single-shot imaging through scattering layers and around corners via speckle correlations," Nature Photonics 8, 784-790 (Aug. 2014).

[7] Duarte, M., Davenport, M., Takhar, D., Laska, J., Kelly, K., and Baraniuk, R., "Single-Pixel Imaging via Compressive Sampling," IEEE Signal Processing Magazine 25, 83-91 (Mar. 2008).

[8] Tajahuerce, E., Durán, V., Clemente, P., Irles, E., Soldevila, F., Andrés, P., and Lancis, J., "Image transmission through dynamic scattering media by single-pixel photodetection," Optics Express 22, 16945 (July 2014).

[9] Marquez, G., Wang, L. V., Lin, S. P., Schwartz, J. a., and Thomsen, S. L., "Anisotropy in the absorption and scattering spectra of chicken breast tissue.," Applied optics 37, 798-804 (Feb. 1998).

[10] Candes, E. and Wakin, M., "An Introduction To Compressive Sampling," IEEE Signal Processing Magazine 25, 21-30 (Mar. 2008).

[11] Soldevila, F., Irles, E., Durán, V., Clemente, P., Fernández-Alonso, M., Tajahuerce, E., and Lancis, J., "Single-pixel polarimetric imaging spectrometer by compressive sensing," Applied Physics B 113, 551-558 (May 2013). 